\title{
Mapping Effective Field Theory to Multifractal Geometry
}

\author{
Ervin Goldfain \\ Research Scholar, Ronin Institute, Montclair, New Jersey 07043 \\ Email: ervin.goldfain@ronininstitute.org
}

\begin{abstract}
Fractals and multifractals are well-known trademarks of nonlinear dynamics and classical chaos. The goal of this work is to tentatively uncover the unforeseen path from multifractals and selfsimilarity to the framework of effective field theory (EFT). An intriguing finding is that the partition function of multifractal geometry includes a signature analogous to that of gravitational interaction. Our results also suggest that multifractal geometry may offer insights into the non-renormalizable interactions presumed to develop beyond the Standard Model scale.
\end{abstract}

Key words: deterministic chaos, multifractals, effective field theory, Lyapunov exponents, Renormalization Group, selfsimilarity.

\section{Introduction}

There is a vast panorama of objects and processes in Nature that exhibit self-similarity, either as "shape invariance" under scaling operations or invariance under scaling of variables defining a system. Examples include sets of fractional dimensions (fractals and multifractals), Levy flights and random walks, fluid turbulence, the geometry of quantum mechanical paths, anomalous diffusion, non-differentiable functions and fractional operators, percolation and crystal growth, self-organized criticality and so on. As defining property of nonlinear dynamics, self-similarity has emerged as common denominator of 
many theoretical frameworks, from the mathematics of chaos and complexity to critical behavior and the Renormalization Group approach to Quantum Field Theory (QFT) [8]. The concepts of continuous dimension and entropy play a pivotal role in the analysis of selfsimilarity. Unlike simple fractals, multifractals are selfsimilar structures endowed with multiple dimensions and are naturally fit to describe the long-run evolution of chaotic phenomena. In particular,

- Nonlinear dynamical systems and iterated maps can generate multifractals by fragmentation of the phase space. The crux of this observation is that both statistical behavior and fragmentation of the phase-space follow from strictly deterministic equations of motion, with no apriori assumptions about randomness and probability distributions [4].

- The long-term chaotic orbits of many dynamical systems are confined to invariant sets called strange attractors, whose characterization requires the language of multifractal geometry.

- Fully developed chaos is, in fact, a faithful replica of equilibrium statistical mechanics. It can be shown that dynamics on a strange attractor displays thermodynamic-like behavior consistent with ergodicity, fluctuation-dissipation theorem, and invariant probability distributions $[1-2,4-5,8]$.

The goal of this work is to tentatively uncover the step-by-step route from multifractals and selfsimilarity to effective field theory (EFT). The paper is organized in the following way: next section is a brief pedagogical introduction to nonlinear maps and Lyapunov stability, section 3 elaborates on the correspondence between the partition function of multifractal geometry and its counterpart of classical Thermodynamics. Drawing from 
the link between Lyapunov exponents and the Gaussian curvature of geodesic trajectories, section 4 argues that the partition function of multifractal geometry includes a signature analogous to that of gravitational interaction. Section 5 suggests that multifractal geometry may offer insights into the hypothetical non-renormalizable interactions beyond the Standard Model (SM) scale. Concluding remarks are outlined in the last section.

We caution the reader on the provisional nature of this early investigation. Further analysis and independent evaluation are needed to refute, confirm, or develop these lines of reasoning and determine their long-term viability.

\section{Nonlinear maps and Lyapunov exponents}

A hallmark feature of chaotic dynamics is sensitivity to initial conditions, which leads to the exponential instability of nearby phase-space trajectories. The separation between adjacent trajectories grows exponentially in time according to

$$
|\delta x(t)| \sim|\delta x(0)| \exp (\lambda t)
$$

for $\lambda>0$. To fix ideas, consider a one-dimensional nonlinear system whose time evolution is described by the iterated map

$$
z_{n+1}=f\left(z_{n}\right)
$$

where the iterates $z_{n}$ are confined to a bounded interval $\left[z_{\min }, z_{\max }\right]$ as $n \rightarrow \infty$. The phase space of (2) is defined by the axis containing the set of $z$ values. Let $z_{0} \rightarrow z_{1} \rightarrow z_{2} \rightarrow \ldots$ 
represent a trajectory starting from the initial point $z_{0}$. Linearizing the map about this trajectory for a nearby initial point $z_{0}+\delta z_{0}$ implies that

$$
z_{n}+\delta z_{n}=f\left(z_{n-1}+\delta z_{n-1}\right) \approx f\left(z_{n-1}\right)+f^{\prime}\left(z_{n-1}\right) \delta z_{n-1}+\ldots
$$

By these arguments, the equation describing the linearized error propagation reads [4]

$$
\delta z_{n} \cong f^{\prime}\left(z_{n-1}\right) \delta z_{n-1}
$$

Labelling the initial separation of two nearby trajectories by $\delta z_{0}$, the closed form solution of (4) can be presented as

$$
\delta z_{n} \cong \prod_{i=0}^{n-1} f^{\prime}\left(z_{i}\right) \delta z_{0}
$$

where $z_{n}=f^{(n)}\left(z_{0}\right)$ is the $n^{\text {th }}$ iterate of (2) starting from $z_{0}$. Therefore,

$$
\left|\delta z_{n}\right| \cong\left|\delta z_{0}\right|\left|f^{(n)^{\prime}}\left(z_{0}\right)\right|
$$

By (1), (6) leads to the following definition of the Lyapunov exponent

$$
\begin{gathered}
\left|f^{(n)^{\prime}}\left(z_{0}\right)\right|=\exp (\lambda n) \Rightarrow \lambda=\frac{1}{n} \ln \left|f^{(n)^{\prime}}\left(z_{0}\right)\right| \\
\delta z_{n} \cong \delta z_{0} \exp (n \lambda)
\end{gathered}
$$

On the other hand, by (5), one can write 


$$
\frac{\delta z_{n}}{\delta z_{0}}=\frac{\delta z_{n}}{\delta z_{n-1}} \frac{\delta z_{n-1}}{\delta z_{n-2}} \ldots \frac{\delta z_{1}}{\delta z_{0}} \Rightarrow \ln \frac{\left|\delta z_{n}\right|}{\left|\delta z_{0}\right|}=\sum_{i=0}^{n-1} \ln \left|f^{\prime}\left(z_{i}\right)\right|
$$

Comparative inspection of (6)-(8) in the limit $n>>1$ yields a consolidated expression of the maximal Lyapunov exponent in the form

$$
\lambda=\lim _{n \rightarrow \infty} \frac{1}{n} \sum_{i=0}^{n-1} \ln \left|f^{\prime}\left(z_{i}\right)\right|=\frac{1}{n} \ln \left|f^{(n)^{\prime}}\left(z_{0}\right)\right|
$$

where

$$
\lambda=\lim _{n \rightarrow \infty} \sum_{i=0}^{n-1} \lambda_{i}
$$

Similar arguments apply to the Lyapunov exponents of higher dimensional maps. Consider, for example, a two-dimensional map given by

$$
x_{n+1}=f\left(x_{n}, y_{n}\right) ; y_{n+1}=g\left(x_{n}, y_{n}\right)
$$

It can be shown that an area element $\Delta a_{n}$ of the phase space defined by $\left(x_{n+1}, x_{n}\right)$ and $\left(y_{n+1}, y_{n}\right)$ evolves as

$$
\Delta a_{n}=\exp \left[n\left(\lambda_{1}+\lambda_{2}\right)\right] \Delta a_{0}
$$

The condition $\lambda_{1}+\lambda_{2}=0$ specifies regular motion in phase space and an area preserving (conservative) map, whereas $\lambda_{1}+\lambda_{2}<0$ describes dissipative motion and a nonconservative map. Deterministic chaos typically requires a dissipative map, along with 
one positive Lyapunov exponent $\lambda_{i}>0$, and generates fragmentation of the phase-space and the onset of strange attractors in the limit $n>>1$.

All these considerations point out that the Lyapunov exponents for any given map determine the stability attributes of its trajectory.

\section{Multifractal geometry as analog of classical Thermodynamics}

A remarkable property of non-invertible maps of the type (2) is that, when $n>>1$, consecutive iterations $z_{n+1}=f\left(z_{n}\right)=f^{(2)}\left(z_{n-1}\right)=\ldots$ produce a partition of the phase-space in $N_{n}$ disjoint intervals of relative lengths $r_{i}=l_{i} / L$, where $L$ is the span of the interval $\left[z_{\min }, z_{\max }\right][1,4]$. In geometric terms, it is customary to refer to this partition as a multifractal set. In the context of multifractal sets, a key concept is the generating function defined as $[4,6]$

$$
\Gamma(q)=\sum_{i=1}^{N_{n}} p_{i}^{q} r_{i}^{\tau(q)}
$$

subject to the normalization condition

$$
\sum_{i=1}^{N_{n}} p_{i}=1
$$

Here, $p_{i}$ is the relative frequency with which the iterated map falls in the $i^{\text {th }}$ interval of the phase space, while $q$ and $\tau(q)$ are continuous scaling exponents $(-\infty<q<\infty)$. It can be shown that the generating function (12) stays invariant to interval rescaling and converges to unity if (and only if) $[1,4,6]$

\section{6 | P a g e}




$$
\Gamma(q) \rightarrow 1 \Leftrightarrow \tau(q)=(1-q) D_{q}
$$

where $D_{q}$ labels the so-called Rényi entropy (or generalized dimension) of multifractal geometry generated by (12).

For an even distribution of iterates $\left(p_{i}=p\right)$ and $n>>1$, the generating function (12) translates to the condition (16) below, namely [4]

$$
\begin{gathered}
p_{i}^{q}=p^{q} \propto\left(1 / N_{n}\right)^{q} \\
N_{n}^{q}=\sum_{i=1}^{N_{n}} r_{i}^{\tau(q)}
\end{gathered}
$$

A closer look at (16) suggests that the even distribution of iterates in the long-time limit $n>>1$ echoes the partition function of Thermodynamics. As stated in the Introduction, this observation is not all that surprising on account of the analogy between fully developed chaos and equilibrium statistical physics. One can thus formally treat (16) as a multifractal partition function as in

$$
Z_{n}(\tau)=\sum_{i=1}^{N_{n}} r_{i}^{\tau(q)}
$$

By the error propagation equation (6)-(7), it is reasonable to expect that the accumulated error satisfies

$$
\delta z_{n} \cong \exp \left(n \lambda_{i}\right) \delta z_{i}
$$

Here, $\lambda_{i}$ is the Lyapunov exponent of the map $z_{n+1}=f\left(z_{n}\right)$ given by (9), namely 


$$
\lambda_{i}=\frac{1}{n} \ln \left|f^{(n)^{\prime}}\left(z_{i}\right)\right|
$$

The so-called backward map iteration starts from the whole phase space $\left[z_{\min }, z_{\max }\right]$ and partitions it in a distribution of disjoint intervals, a process that is formally equivalent to coarse graining of the phase space [4]. It is intuitively clear that coarse graining by backward iteration mirrors the error propagation equation (18), which describes the progressive growth of separation between nearby trajectories. As a result, setting the maximal propagation error to unity, and performing the identification [4]

$$
\delta z_{i}=r_{i}^{(n)}
$$

leads to

$$
r_{i}^{(n)} \cong \exp \left(-n \lambda_{i}\right)
$$

Direct substitution of (21) in (17) gives

$$
Z_{n}(\tau)=\sum_{i=1}^{N_{n}} \exp \left(-n \tau \lambda_{i}\right)
$$

The multifractal partition function (22) may be alternatively expressed as a sum over Lyapunov exponents, as in

$$
Z_{n}(\tau)=\sum_{\lambda} N(\lambda) \exp (-n \tau \lambda)
$$


where $N(\lambda)$ is the number of intervals with the same exponent $\lambda$. Invoking the correspondence between the exponent $\tau(q)$ and the free energy of a thermodynamic system $F(\beta)$, where $q=\beta=T^{-1}$, yields [9]

$$
\tau(q)=q F=\beta F(\beta)
$$

Accordingly, (23) turns into

$$
Z_{n}(\beta)=\sum_{\lambda} N(\lambda) \exp [-n \lambda \beta F(\beta)]
$$

or

$$
Z_{n, \lambda}(\beta)=\sum_{\lambda} N(\lambda) \exp \left[-\beta F_{n, \lambda}(\beta)\right]
$$

where

$$
F_{n, \lambda}(\beta)=n \lambda F(\beta)
$$

It is apparent that (26) generalizes the canonical partition function of Thermodynamics, by including in its expression the Lyapunov exponents $\lambda$ and the number of map iterations $n$. A glance at (1) and (7) shows that a vanishing $\lambda$ signals conservative dynamics, whereby the trajectory error $\delta z_{n}$ assumes a stationary value $\delta z_{n}=\delta z_{0}$. This observation hints that, in order to cast (26) in a form more suggestive of its analogy with Thermodynamics, one may use the convenient transformation

$$
\bar{\lambda}=1+\lambda
$$


so that $\lambda=0$ amounts to $\bar{\lambda}=1$. Assuming that there is only one Lyapunov exponent and substituting (28) into (26) yields

$$
Z_{n}(\beta) \propto \exp \left[-\beta F_{n, \bar{\lambda}}(\beta)\right]
$$

whose thermodynamic analog corresponds to $n>>1, \bar{\lambda}=1$ and is given by

$$
Z_{t h}=Z_{\infty}(\beta) \propto \exp \left[-\beta F_{\infty, 1}(\beta)\right]
$$

\section{Non-Euclidean metric and multifractal geometry}

The goal of next two sections is to bridge the gap between multifractal geometry and the framework of effective field theory (EFT), with emphasis on General Relativity and the Standard Model of particle physics (SM).

Let us first recall that the inherent sensitivity of geodesics to initial conditions connects their Lyapunov exponents $\left(\lambda_{i}\right)$ to the local Gaussian curvature $(K)$ via $[3,6]$

$$
\lambda_{i} \Leftrightarrow \sqrt{K}
$$

It follows from (31) that the $K$ represents a local measure of geodesic instability. At the same time, the concept of Kolmogorov entropy $\left(S_{K}\right)$ quantifies the amount of information lost or gained in the flow towards chaos of generic nonlinear dynamical systems $[2-3,6,10]$. It can be shown that $S_{K}$ relates to the spectrum of Lyapunov exponents as in [2-3] 


$$
S_{K}=\int_{\Pi} \sum_{\left|\lambda_{i}\right|>1} \log \left|\lambda_{i}\right| d \mu
$$

where the integral is taken over the phase space $\Pi$, whose differential measure is $d \mu$. Taken together, (29), (31) and (32) hint that there is an intriguing relationship between the partition function of multifractal geometry and the non-Euclidean metric of General Relativity. In particular, the Lyapunov exponents entering (29) describe effects analogous to those induced by gravitation, from which they decouple in the corresponding limit of flat spacetime, where $K \rightarrow 0$ together with $\lambda_{i} \rightarrow 0$ and $\bar{\lambda}_{i} \rightarrow 1$.

By (6) and (7), the only setting consistent with $\lambda_{i} \rightarrow 0$ and $K \rightarrow 0$ is $\delta z_{n}=\delta z=$ const., which means a stationary deviation from initial conditions. It follows from these considerations that conditions akin to Euclidean spacetime and equilibrium Thermodynamics are recovered in the asymptotic regime defined by $\lambda_{i} \rightarrow 0$ and $\bar{\lambda}_{i} \rightarrow 1$. These results can be symbolically summarized as follows

$$
\lambda=0 \Leftrightarrow K=0 \Leftrightarrow Z_{t h}(\beta) \propto \exp \left[-\beta F_{\infty, 1}(\beta)\right]
$$

Appealing to (11), one finds that (33) matches the concept of non-conservative maps $\left(\lambda_{i} \neq 0\right)$ with the nonvanishing curvature of General Relativity. This result is consistent with the fact that General Relativity fails to comply with the global conservation of the energy-momentum tensor. Conversely, strictly conservative dynamics in the Lyapunov sense $\left(\lambda_{i}=0\right)$ echoes the settings of both Thermodynamics and field theory in Euclidean spacetime. 
Given the known analogy between Thermodynamics and Euclidean Quantum Field Theory (QFT) [11], we believe that these findings point to a hypothetical connection between QFT and General Relativity and a possible path towards unification based upon multifractal geometry.

\section{Multifractals and physics beyond the Standard Model}

Moving onto the EFT, we recall the expression of the effective field Lagrangian in $d$ spacetime dimensions [12]

$$
L_{e f f}=\sum_{k} c_{k} O_{k}
$$

where $\left\{O_{k}\right\}$ are local operators compliant with the symmetries of the theory and built from fields describing the low-energy sector. By analogy with the generating function of multifractal analysis (12), we can write

$$
\Gamma_{\text {eff }}=\frac{1}{L_{\text {eff }}} \sum_{k} c_{k} O_{k}
$$

and demand that (35) converges to unity if the mass dimensions of coefficients $c_{k}$ and of operators $O_{k}$ satisfy (14). Since the mass dimension of the Lagrangian is equal to $d$, one has

$$
\left[L_{e f f}\right]=M^{d} \Rightarrow \sum_{k} \frac{c_{k}}{M^{d-\Delta_{k}}} \frac{O_{k}}{M^{\Delta_{k}}}=1
$$

in which $\Delta_{i}$ stands for the mass dimension of operator $O_{i}$. Therefore, 


$$
\sum_{k} \frac{c_{k}}{M^{d-\Delta_{k}}} \frac{O_{k}}{M^{\Delta_{k}}}=\sum_{k}\left(\frac{c_{k}^{1 /\left(d-\Delta_{k}\right)}}{M}\right)^{d-\Delta_{k}}\left(\frac{O_{k}^{1 / \Delta_{k}}}{M}\right)^{\Delta_{k}}=1
$$

Relation (37) may be mapped to (12)-(14) with the identification

$$
\left(\frac{c_{k}^{1 /\left(d-\Delta_{k}\right)}}{M}\right)^{d-\Delta_{k}} \Rightarrow p_{i}^{q}
$$

in which

$$
\sum_{k} \frac{c_{k}^{1 /\left(d-\Delta_{k}\right)}}{M}=1
$$

and

$$
\left(\frac{O_{k}^{1 / \Delta_{k}}}{M}\right)^{\Delta_{k}} \Rightarrow r_{i}^{\tau(q)}
$$

Besides (39), we proceed with the following assumptions:

A1) The effective Lagrangian (34) contains individual groups of terms having the same mass dimension $\Delta_{l}(l<k)$. To avoid cluttering the notation, we use a single index for labeling these terms, that is, $l=k$.

A2) Each operator $O_{k}$ is the product of operators $O_{k}=O_{1} O_{2} \ldots O_{k}$.

Let the overall mass dimension of (34) be

$$
\Delta(d)=\sum_{k} \Delta_{k}(d)
$$


By (14) and (37)-(41), we obtain the following expression of the generalized dimension of the dynamics described by (34)

$$
\tau(q)=(1-q) D_{q} \Leftrightarrow D_{e f f}(d)=\left|\frac{1}{Q(d)+1}\right|
$$

where

$$
Q(d)=\frac{1-d}{\Delta(d)}
$$

It is instructive to note that, setting the mass dimension of the effective Lagrangian to equal the spacetime dimension, forces the generalized dimension (42) to also be equal to $d$, i.e.

$$
\Delta(d)=d \Rightarrow D_{e f f}(d)=d
$$

The Standard Model (SM) Lagrangian in four dimensional spacetime offers a straightforward example of (43), as the characteristic mass dimension of its interactions is $D_{S M}=d=4$. Non-renormalizable interactions above the SM scale $\left(\Lambda>M_{S M}\right)$ add high order terms to the effective Lagrangian as in [12]

$$
L_{e f f}=L_{S M}+\sum_{k} \frac{L_{k}}{\Lambda^{k-4}}, \quad k \geq 5
$$

It is seen from (41), (42) and (44) that the contribution of high order terms $(k \geq 5)$ yields a larger overall dimension $\Delta(d=4)$, which leads in turn to a lower Rényi entropy $D_{e f f}(d=4)$. This speculative scenario hints that non-renormalizable interactions above 
the SM scale may generate self-organized critical behavior accompanied by a corresponding drop in entropy.

\section{Conclusions and outlook}

This tentative analysis has argued that there is an unexplored path linking multifractal geometry to both relativistic physics and the low-energy framework of the Standard Model. A thought-provoking assertion is that the partition function of multifractal geometry includes effects akin to classical gravity. Results also suggest that multifractal geometry may shed light onto the regime of non-renormalizable interactions presumed to occur beyond the Standard Model scale.

We believe that further studies are needed to substantiate, refute, or develop these findings along the following directions:

1. Ref. [6] has found that the four dimensionality of classical spacetime emerges from the Rényi entropy of geodesic trajectories for $q=1 / 2$, as given by $D_{1 / 2}=4$. It is known that the familiar Hausdorff dimension $D_{H}=D_{0}$ corresponds to $q=0$. Since $q=T^{-1}$ by (24), the temperature analog at this value is $T=1 / q \rightarrow \infty$, indicative of the Planckian regime of the Big Bang singularity, where the concept of GR metric likely breaks down. As the Universe expands and cools off, the temperature drops and $q$ goes up from zero to $q=1 / 2$, which explains why $D_{1 / 2}=4$ at $q=1 / 2$. Can this assertion be corroborated with additional arguments stemming from multifractal geometry and the transition to chaos of nonlinear dynamics? 
2. Following [17], how is the generalized dimension (42a) linked to non-integrability and the transition to chaos beyond the Standard Model scale? Is it conceivable that Dark Matter is a hidden manifestation of the Rényi entropy $D_{q}$ defined in (14) and its dimensional condensation, as pointed out in [18]?

\section{References}

1. https://www.researchgate.net/publication/272400210

2. Tabor, M. Chaos and Integrability in Nonlinear Dynamics: An Introduction; Wiley: New York, NY, USA, 1989.

3. Gutzwiller, M. C. Chaos in Classical and Quantum Mechanics; Springer-Verlag: New York, NY USA, 1990.

4. McCauley J. L. Chaos, Dynamics, and Fractals: An algorithmic approach to deterministic chaos; Cambridge Univ. Press: Cambridge, 1994.

5. Beck, C. and Schlögl R. Thermodynamics of chaotic systems-an introduction; Cambridge Univ. Press: Cambridge, 1993.

6. https://www.researchgate.net/publication/351068496

7. Creswick R.J. et al Introduction to Renormalization Group Methods in Physics; John Wiley \& Sons: New York, NY, USA, 1992.

8. Goldfain, E. On the Role of Self-similarity in the Dynamics of Effective Field Theory. Preprints 2020, 2020120012 (DOI: 10.20944/preprints 202012.0012.v1). 
9. Goldfain, E. Emergence of Lagrangian Field Theory from Self-Organized Criticality. Preprints 2020, 2020110518 (DOI: 10.20944/preprints 202011.0518.v1).

10. https://www.researchgate.net/publication/336047781

11. Zinn-Justin, J. Quantum Field Theory and Critical Phenomena; Clarendon Press: Oxford, 2004.

12. Donoghue, J. F. et al Dynamics of the Standard Model; Cambridge Univ. Press: Cambridge, 1994.

17. Goldfain, E. Non-Integrable Dynamics and Physics Beyond the Standard Model. Preprints 2021, 2021020564 (DOI: 10.20944/preprints 202102.0564.v2).

18. https://www.researchgate.net/publication/343426202 